\title{
Generating randomized trial evidence to optimize treatment in the COVID-19 pandemic
}

\author{
Matthew P. Cheng MDCM, Todd C. Lee MD MPH, Darrell H.S. Tan MD PhD, Srinivas Murthy MDCM MHSc
}

Cite as: CMAJ 2020 April 14;192:E405-7. doi: 10.1503/cmaj.200438; early-released March 26, 2020

W hile aggressive mitigation strategies are being implemented to slow the spread of severe acute respiratory syndrome coronavirus 2 (SARS-CoV-2), we must also strive to optimize clinical outcomes for those infected and prevent transmission among their close contacts. As the number of severely ill patients with coronavirus disease 2019 (COVID-19) continues to increase, we must learn as much as possible about this disease, as quickly as possible, to inform patient care. Fortunately, the Canadian clinical research community has already mobilized to ensure a coordinated effort of randomized controlled trials (RCTs) on treatment, prophylaxis and vaccines. We discuss why and how, in collaboration with global colleagues, Canadian researchers are poised to generate high-quality evidence that may improve outcomes for all Canadians and patients worldwide.

When faced with an unknown and frightening disease such as COVID-19, and given concerns over a potentially high case fatality rate, some clinicians and patients will feel strongly compelled to try unproven therapies based on theory, in vitro data, animal models, clinical anecdotes, observational studies confounded by severity, and uncontrolled or underpowered trials that may later be shown to be misleading. ${ }^{1-3}$ For example, during the novel influenza A (H1N1) viral pandemic in 2009, countries stockpiled and used oseltamivir extensively, at great expense, based on data of suboptimal quality showing a potential benefit in patients admitted to hospital. ${ }^{4}$ Even now, because there has been no RCT, it is not definitively known whether oseltamivir is efficacious for preventing or treating the complications of influenza in admitted patients.

In the COVID-19 pandemic, experimental medications that are not currently approved for any indication in any country have already been widely used outside of clinical trial protocols. ${ }^{5}$ But there is a strong ethical and clinical argument for replacing such "random" care with randomized care, in which patients are routinely randomly assigned to the most promising available option(s) or to control arm(s), so that evidence regarding the safest, most effective therapies may be generated in the shortest possible time. This means that although earlier patients may receive our best-guess treatments, subsequent patients can receive evidence-based therapies and be spared from harm.

\section{KEY POINTS}

- When faced with a new and therefore frightening disease such as coronavirus disease 2019 (COVID-19), and given concerns over a potentially high case fatality rate, clinicians and patients will be strongly compelled to try unproven therapies.

- We must avoid "random" care and ensure that patients are routinely randomly assigned to the most promising available treatment(s) or to control arm(s), as suggested by the World Health Organization, so that evidence regarding the safest, most effective therapies is generated in the shortest possible time.

- The World Health Organization has prioritized several medications for further study in COVID-19, including lopinavirritonavir, remdesivir and (hydroxy)chloroquine.

- Canada's clinical research infrastructure is being scaled quickly to meet the challenge, with regulatory bodies acting swiftly to facilitate initiation of Canada's first COVID-19 trials that form part of a global research effort to study treatment and prevention strategies.

The World Health Organization (WHO) has prioritized several medications for further study in COVID-19 - based on in vitro data, available safety data and biologic plausibility - and has recommended that these be evaluated in the context of a clinical trial (www.who.int/blueprint/priority-diseases/key-action/novel -coronavirus/en). These agents currently include lopinavirritonavir, remdesivir and (hydroxy)chloroquine; as new data emerge, others may be added. With no proven effective treatments for COVID-19, showing that any drug has a net clinical benefit is a public health priority.

More than 500 clinical trials are already registered on the WHO International Clinical Trials Registry Platform, many of which are actively recruiting (https://apps.who.int/trialsearch). This is impressive, but also concerning. With so many different trials, there are risks of wasteful duplication, competition for the same participants, and the potential for underpowered studies to lead to either the premature rejection of promising drugs ${ }^{6}$ or premature adoption into standard of care. These risks must be prevented by a commitment to collaboration among research groups worldwide. 
We believe that Canada needs to ensure the necessary infrastructure is in place to support enrolment of patients with COVID-19 in treatment trials, making it simple for all clinicians to think about whether every patient they see is eligible for randomization and, if they are, to approach the patient about enrolment in clinical trials that are being transparently conducted in communication with global partners. This strategy would allow clinicians to be satisfied that they are doing everything possible for their severely ill patients while contributing to new knowledge. Patients agree with this approach, as shown by a number of studies conducted before the current pandemic. ${ }^{7,8}$

Canada's clinical research infrastructure is being scaled quickly to meet these scientific and ethical imperatives, similar to trials that were conducted during the Ebola epidemic. ${ }^{9}$ The Canadian Institutes of Health Research and its partners launched a rapid research funding opportunity on COVID-19 in February 2020, completing applications, submissions, peer review and initial funding decisions within 18 days. Regulatory bodies have acted swiftly to facilitate initiation of Canada's first COVID-19 trials.

CATCO (Canadian Treatments for COVID-19), a large treatment trial in Canada, will be part of the WHO Solidarity Protocol, a global protocol to evaluate the best available agents for patients admitted to hospital. The WHO protocol has a goal of enrolling thousands of patients worldwide to achieve the most precise possible estimate of treatment effect of high-priority agents in the shortest time possible. Canada will start by evaluating lopinavir-ritonavir, compared with optimized supportive care, and add new therapies as data emerge. Given that effect sizes are not known, accurate sample-size calculation is impossible. Ethics approval for the CATCO trial was granted within 5 working days of protocol submission in Ontario and 10 in British Columbia, approvals in Quebec and Alberta are forthcoming, and Health Canada gave approval within 3 working days of first submission. This unprecedented institutional support for these efforts must be applauded.

REMAP-CAP (A Randomised, Embedded, Multi-factorial, Adaptive Platform Trial for Community-Acquired Pneumonia) is an adaptive platform trial established before the COVID-19 pandemic, examining a variety of interventions in critically ill patients with pneumonia. ${ }^{10}$ In Canada and 13 other countries, the trial has now pivoted to include interventions specific to COVID-19 and supportive interventions, including macrolides, steroids and interferon- $\beta-1 \mathrm{a}$, with antiviral administration. Both the CATCO and the REMAP-CAP trials are studying effects in the severely ill, and recruitment is coordinated between the two. Stopping rules have been modified, primary outcomes have been altered and subsequent inclusion of other agents is planned to more rapidly achieve an evidence base during the pandemic.

Evidence on how to prevent new infections will also be critical, especially among older adults in care facilities and health care workers, in whom maintaining health and morale is vital. The CORIPREV LR (COVID-19 Ring-based Prevention Trial with Lopinavir/Ritonavir) will evaluate the efficacy of lopinavir-ritonavir as postexposure prophylaxis in preventing microbiologically confirmed infection. This trial will use an approach adapted from a cluster randomized trial conducted during the 2013-2016 Ebola epidemic in West Africa; ${ }^{11}$ researchers will define "rings" of exposed contacts around infected cases and randomly assign them to prophylaxis or usual preventive measures. In CORIPREV LR, 220 rings will be randomly assigned to either lopinavir-ritonavir or no intervention, with participants in both arms undergoing daily symptom self-monitoring and weekly screening tests. Ring vaccination was key to the successful eradication of smallpox. ${ }^{12}$ The CORIPREV LR trial infrastructure can later be harnessed to rapidly evaluate the efficacy of vaccines, when they become available.

Challenges to RCTs during the COVID-19 pandemic include maintaining adequate funding, rapid scale-up and limiting researchers' risk of acquiring infection. Novel strategies may be needed for obtaining informed consent, harnessing video and other remote technologies. Data entry must be simplified, prioritizing information of greatest relevance. Along these lines, in conjunction with lead investigators in Minnesota, the COVID19 Postexposure Prophylaxis RCT - Canada will allow health care workers and community-dwelling adults with high-risk exposures or early symptomatic disease to screen themselves electronically, receive their assigned study drug (hydroxychloroquine v. placebo) via overnight courier, and follow up by self-report. Outcomes include symptomatic disease for initially asymptomatic participants and severe disease for those who were symptomatic. A pooled data set allows the Canadian results to be combined with the international results in near real time to achieve an estimated sample size of 1500 per cohort.

As the pandemic evolves, the temptation to use unproven medications will be tremendous, but Canadian clinicians must maintain a commitment to the rigorous gathering of scientific evidence, in collaboration with global colleagues, so that we may quickly improve outcomes for all patients with COVID-19 worldwide. A community-sourced registry of known Canadian trials can be found at canada-covid.idtrials.com and will be updated as more information becomes available.

\section{References}

1. Wang M, Cao R, Zhang L, et al. Remdesivir and chloroquine effectively inhibit the recently emerged novel coronavirus (2019-nCoV) in vitro. Cell Res 2020;30:269-71.

2. Xu Z, Shi L, Wang Y, et al. Pathological findings of COVID-19 associated with acute respiratory distress syndrome. Lancet Respir Med 2020 Feb. 18 [Epub ahead of print]. doi: 10.1016/S2213-2600(20)30076-X.

3. Herrera-Perez D, Haslam A, Crain T, et al. A comprehensive review of randomized clinical trials in three medical journals reveals 396 medical reversals. eLife 2019;8:e45183. doi: 10.7554/eLife.45183.

4. Dyer O. What did we learn from Tamiflu? BMJ 2020;368:m626.

5. Lai CC, Shih TP, Ko WC, et al. Severe acute respiratory syndrome coronavirus 2 (SARS-CoV-2) and coronavirus disease-2019 (COVID-19): the epidemic and the challenges. Int J Antimicrob Agents 2020;55:105924.

6. Cao B, Wang Y, Wen D, et al. A trial of lopinavir-ritonavir in adults hospitalized with severe COVID-19. N Engl J Med 2020 Mar. 18 [Epub ahead of print]. doi: 10.1056/NEJMoa2001282.

7. Gobat NH, Gal M, Butler CC, et al. Talking to the people that really matter about their participation in pandemic clinical research: a qualitative study in four European countries. Health Expect 2018;21:387-95. 
8. Gobat N, Butler CC, Mollison J, et al. What the public think about participation in medical research during an influenza pandemic: an international crosssectional survey. Public Health 2019;177:80-94.

9. Mulangu S, Dodd LE, Davey RT Jr., et al.; PALM Consortium Study Team. A randomized, controlled trial of Ebola virus disease therapeutics. N Engl J Med 2019;381:2293-303.

10. REMAP-CAP: A Randomized, Embedded, Multi-factorial, Adaptive Platform Trial for Community-Acquired Pneumonia [main page]. Available: www. remapcap.org (accessed 2020 Mar. 3).
11. Henao-Restrepo AM, Camacho A, Longini IM, et al. Efficacy and effectiveness of an rVSV-vectored vaccine in preventing Ebola virus disease: final results from the Guinea ring vaccination, open-label, cluster-randomised trial (Ebola Ça Suffit!). Lancet 2017;389:505-18.

12. Kretzschmar $M$, van den Hof $S$, Wallinga J, et al. Ring vaccination and smallpox control. Emerg Infect Dis 2004;10:832-41.
Competing interests: Todd Lee receives salary support from the Fonds de recherche du Québec - Santé. Darrell Tan's institution has received investigator-initiated research grants or in-kind support from Gilead, Viiv Healthcare and AbbVie. Darrell Tan is a site principal investigator for clinical trials sponsored by GlaxoSmithKline. Darrell Tan is supported by a Tier 2 Canada Research Chair. Srinivas Murthy's institution has received in-kind support from AbbVie.
This article has not been peer reviewed.

Affiliations: Division of Infectious Diseases (Cheng, Lee), Department of Medicine, and Division of Medical Microbiology (Cheng), Department of Laboratory Medicine, McGill University Health Centre; Clinical Trials Platform (Cheng, Lee), McGill Interdisciplinary Initiative in Infection and Immunity, Montréal, Que.; Division of Infectious Diseases (Tan) and MAP Centre for Urban Health Solutions (Tan),
St. Michael's Hospital; Department of Medicine (Tan), University of Toronto, Toronto, Ont.; Department of Pediatrics (Murthy), University of British Columbia, Vancouver, BC

Contributors: All of the authors drafted and revised the manuscript, gave final approval of the version to be published, and agreed to be accountable for all aspects of the work.

Correspondence to: Srinivas Murthy, srinivas. murthy@cw.bc.ca 